\title{
Range-wide phylogeography and conservation genetics of a narrowly endemic stream salamander, Pachyhynobius shangchengensis (Caudata, Hynobiidae): implications for conservation
}

T. Pan ${ }^{1}$, H. Wang ${ }^{1}$, C.-C. Hu ${ }^{2}$, W.-B. Shi ${ }^{1}$, K. Zhao ${ }^{3}$, X. Huang ${ }^{1}$ and B.-W. Zhang ${ }^{1}$

${ }^{1}$ School of Life Science, Anhui University, Hefei, Anhui, China

${ }^{2}$ School of Life Science, Nanjing Normal University, Nanjing, Jiangsu, China

${ }^{3}$ Department of Life Science, Anqing Teachers College, Anqing, Anhui, China

Corresponding author: B.-W. Zhang

E-mail: zhangbw@ahu.edu.cn

Genet. Mol. Res. 13 (2): 2873-2885 (2014)

Received August 18, 2013

Accepted December 15, 2013

Published February 13, 2014

DOI http://dx.doi.org/10.4238/2014.February.13.9 
northern populations (JGT, KHJ). Significant population differentiation $\left(F_{\mathrm{ST}}\right)$ was detected among all populations. Among the four populations, historical demographic analyses (e.g., the g parameter, the Tajima D test, and the Fu Fs test) did not reveal definite information on population expansion except for the BYM population, which had undergone a strong population expansion event. Based on the analysis of a Bayesian skyline plot, the total population underwent a significant population fluctuation around 20 kya. This may have been triggered by the end of the last glacial maximum. In conclusion, the existence of three evolutionarily significant units (BMY-TM, KHJ, and JGT) and four management units (BMY, TM, KHJ, and JGT) is supported by our study.

Key words: Pachyhynobius shangchengensis; Genetic structure; Mitochondrial DNA control region; Phylogeography;

Population demography

\section{INTRODUCTION}

During Pleistocene glacial cycles, climatic changes profoundly affected species distributions. In the temperate zones, many species have undergone extreme changes, and even in the non-glacial areas some species have suffered from climatic fluctuations. In montane regions, some species expand or contract their habitat along an elevation gradient in order to adapt to changing climatic conditions (Hewitt, 2004; DeChaine and Martin, 2005). As a result, adjacent populations become isolated or associated with different populations during this process; periods of isolation lead to genetic divergence, whereas periods of association encourage dispersal and gene flow (Wiens, 2004). Repeated isolation and connectivity of adjacent populations has had a complex effect on the genetic structure of populations (Hewitt, 2004).

The availability of adequate biological information is the foundation of successful conservation and restoration of any declining species (Lind et al., 2011). For effective management and restoration, species conservation increasingly relies on genetic information, especially for defining management units. Several management-oriented categories have been created for conservation work, such as evolutionary significant units, distinct population segments, and management units (De Guia and Saitoh, 2007). These categories have contributed significantly to the conservation of endangered species (Palsbøll et al., 2007). In recent years, in order to construct better management-oriented categories for conservation, considerable attention has been given to phylogeography, molecular phylogenetics, and evolution.

Amphibians are generally more susceptible to environmental change than other animals and their conservation attracts concern all over the world (Murphy et al., 2000). In addition, amphibians also have great value in the field of phylogeny, speciation, phylogeography, and genome evolution. Pachyhynobius shangchengensis is a species narrowly endemic to the Dabie Mountains (Figure 1), which include the counties of Huoshan, Yuexi, Jinzhai, Yingshan and Shangcheng (Zhang et al., 2009). There are four geographically isolated populations: Baimajian/Mingtangshan/Yaoluopin (BMY), Tiantangzhai/Mazongling (TM), Kangwangzhai/Huangbaishan/Jiufengjian (KHJ), and Jingangtai (JGT), listed from south to north. P. shangchengensis are generally found in cool, oxygen-rich streams with slow to moderate flow, and feed on small aquatic invertebrates. However, they are now largely restricted to streams above 500 meters (Fei 
et al., 1985; Cai, 2001). The main reason for this phenomenon may be environmental adaptation and low vagility. Recently, because of deforestation, habitat destruction, and illegal capture, the total population of $P$. shangchengensis has decreased sharply (Cai, 2001; Zhang et al., 2009).

Since the mitochondrial DNA control region (CR) has a high evolutionary rate, it is considered an excellent genetic marker for the mitochondrial genome. In the last few decades, the mtDNA CR has been adopted widely in phylogeographic and genetic structure analyses for different groups of vertebrates (Steinfartz et al., 2000). In molecular ecology studies of caudates, there are many successful examples of its use as a molecular marker, such as in the phylogeographic study of tiger salamanders (Ambystoma tigrinum) (Church et al., 2003) and lineage divergence of Salamandra taxa in Europe (Steinfartz et al., 2000).

Here we report a detailed study of the phylogeography and conservation genetics of $P$. shangchengensis. Our main objectives were as follows: 1) To probe the genetic diversity in narrowly distributed $P$. shangchengensis; 2) To use statistical phylogenetic methods to test whether each geographically isolated mountain population comprises a distinct evolutionary lineage of $P$. shangchengensis; 3) To use statistical demographic methods to test the links between the Pleistocene climatic cycles and the potential lineage divergence of $P$. shangchengensis.

\section{MATERIAL AND METHODS}

\section{Sampling}

We conducted extensive an survey throughout the Dabie Mountains and the interflowing streams to determine the distribution of $P$. shangchengensis and collected 175 individuals in total from the four populations (BYM, 54; TM, 38; KHJ, 36; JGT, 47) from April 2008 to August 2011. Sampling involved capturing $P$. shangchengensis using dip nets, removing the tip of the tail (about $1 \mathrm{~cm}$ ) and releasing the individuals; the tissue samples were preserved in $95 \%$ ethanol.

\section{Amplification and sequencing}

We extracted total DNA using a standard proteinase K/phenol-chloroform protocol (Sambrook et al., 1989). An EasyPure PCR Purification Kit (TransGene) was used to purify each DNA extract. Primers, PshCR-f (5'-CCT GGC TCC CAA AGC CAG A-3') and PshCR-r (5'-GGC ATT TTC AGT GCC ATG-3'), were designed using Primer Premier 5.0 (Clarke and Gorley, 2001), to amplify the locus between nucleotide 15625 and 16390 of the complete mitochondrial genome sequence of $P$. shangchengensis (NCBI accession No. DQ333812.1). Polymerase chain reaction (PCR) was performed using a reaction mixture $(30 \mu \mathrm{L})$ containing $200 \mathrm{ng}$ template DNA, $5 \mu \mathrm{L} \mathrm{10X}$ buffer (including $\mathrm{MgSO}_{4}$ at a final concentration of $2 \mathrm{mM}$ ) $880 \mu \mathrm{M}$ dNTPs, $2.5 \mathrm{U}$ Taq polymerase (Meridian Bioscience, Singapore) and $0.3 \mu \mathrm{M}$ primer (each). Pure water was added to reach the appropriate volume. The amplification protocol included an initial denaturation step of $95^{\circ} \mathrm{C}$ for $5 \mathrm{~min}$; this was followed by cycles of denaturation at $95^{\circ} \mathrm{C}$ for $30 \mathrm{~s}$, primer annealing at $55^{\circ} \mathrm{C}$ for $40 \mathrm{~s}$, and extension at $72^{\circ} \mathrm{C}$ for $1 \mathrm{~min}$, with a final extension at $72^{\circ} \mathrm{C}$ for $10 \mathrm{~min}$. PCR products were purified using an EasyPure PCR Purification Kit (TransGene), and sequenced using the PshCR-f/PshCR-r primers and the BigDye Terminator v3.0 Ready Reaction Cycle Sequencing Kit (Applied Biosystems) following manufacturer instructions on an ABI 370 automated sequencer. 


\section{Sequence data analysis}

Sequences were assembled by Seqman II (DNAStar, Madison, WI, USA) and checked by eye to ensure the accuracy of variable sites identified by the program. The mtDNA CR sequences were aligned using ClustalW (Thompson et al., 1994) and visually checked. Initial sequence comparisons and measures of variability were performed using MEGA 4.0 (Tamura et al., 2007), and unique haplotypes were verified using DAMBE. Nucleotide and haplotype diversity was estimated using DnaSP v5 (Librado and Rozas, 2009).

We used the neighbor-joining method of phylogenetic analysis in PAUP* 4.0 (Swofford, 2003). A homologous sequence of Ranodon sibiricus (GenBank accession No. NC004021) was used as an outgroup (Zhang et al., 2006). Statistical support for the resulting phylogeny was assayed by conducting 1000 nonparametric bootstrap pseudoreplicates, with 100 random-taxonaddition replicates per pseudoreplicate. A median-joining haplotype network was constructed using Network 4.6.1.0 (fluxus-engineering.com; Bandelt et al., 1999) weighing characters equally.

Analysis of molecular variance (AMOVA) was performed to test for differentiation between geographical units; the significance of the $\Phi$-statistics generated was tested by random permutations of sequences among populations. The $\mathrm{P}$ values of the $\Phi$-statistics were calculated based on 1000 nonparametric permutations. The optimal grouping option was identified by maximizing the among-group component $\left(F_{\mathrm{CT}}\right)$ of the overall genetic variance. Meantime pairwise $F_{\mathrm{ST}}$ estimates were computed by the HKY distance. Euclidean distances $(\mathrm{km})$ were calculated using an online calculator (Veness, 2010), based on the latitude and longitude of each population. In addition, Mantel tests were used to assess isolation by distance (IBD) of the populations. The plausibility of an IBD scenario was explicitly tested in the different lineages.

To examine the predictions made by the neutral theory of molecular evolution and for evidence of population expansion events, the Tajima D test performed and the Fu Fs statistical test was calculated in Arlequin (Excoffier et al., 2005). Mismatch distribution analysis was performed in Arlequin to further explore the demographic evolution of $P$. shangchengensis, and the sum of squared deviations and raggedness index (r) between the observed and simulated values were calculated to examine the validity of the sudden population expansion model.

The population dynamics of the phylogeographic lineages of $P$. shangchengensis were simulated using the Bayesian skyline plot (BSP) approach. We used the HKY+G model as recommended by MrModeltest 2.3 (Nylander, 2004) to construct BSPs in BEAST 1.4.8 (Drummond and Rambaut, 2007) for each lineage. The evolutionary rate of the mtDNA CR in the genus Salamandra ranged from 0.68 to $1.5 \%$ per million years (Steinfartz et al., 2000); here we chose an approximate intermediate value, $1 \%$, as the molecular clock standard for BSP construction. These analyses estimated genealogies and model parameters, and were sampled every 1000th iteration for 10 million generations with the first $10 \%$ of samples discarded as burn-in. Data for all populations were plotted and the posterior distribution of effective population size was calculated using Tracer 1.4 (Rambaut and Drummond, 2007).

\section{RESULTS}

\section{Genetic diversity and haplotype distribution}

We obtained complete sequences of the 768-bp mtDNA CR for all the individuals ana- 
lyzed $(\mathrm{N}=175)$. A total of 28 haplotypes (accession Nos. KC254826-KC254853) were found, defined by 37 variable positions, of which 35 were parsimony-informative and 2 were singletons. The range of pairwise distance between individual was $0.001-0.031$. The base composition of the mtDNA CR sequence is $30.8 \% \mathrm{~A}, 14.6 \% \mathrm{G}, 19.3 \% \mathrm{C}$, and $35.4 \% \mathrm{~T}$, so the percentage of $\mathrm{A}$ and $\mathrm{T}(66.2 \%)$ was significantly higher than that of $\mathrm{G}$ and $\mathrm{C}$. The genetic diversity of the four populations is shown in Table 1. Among the four populations, TM showed the highest value for haplotype $(27.03 \%)$ and nucleotide diversity $(\pi=0.0050 \pm 0.0034$; Table 1$)$, thus demonstrating a high genetic diversity. The other populations exhibited a similar level of diversity, except KHJ, which contained just two haplotypes among the 36 individuals sampled (Figure 1).
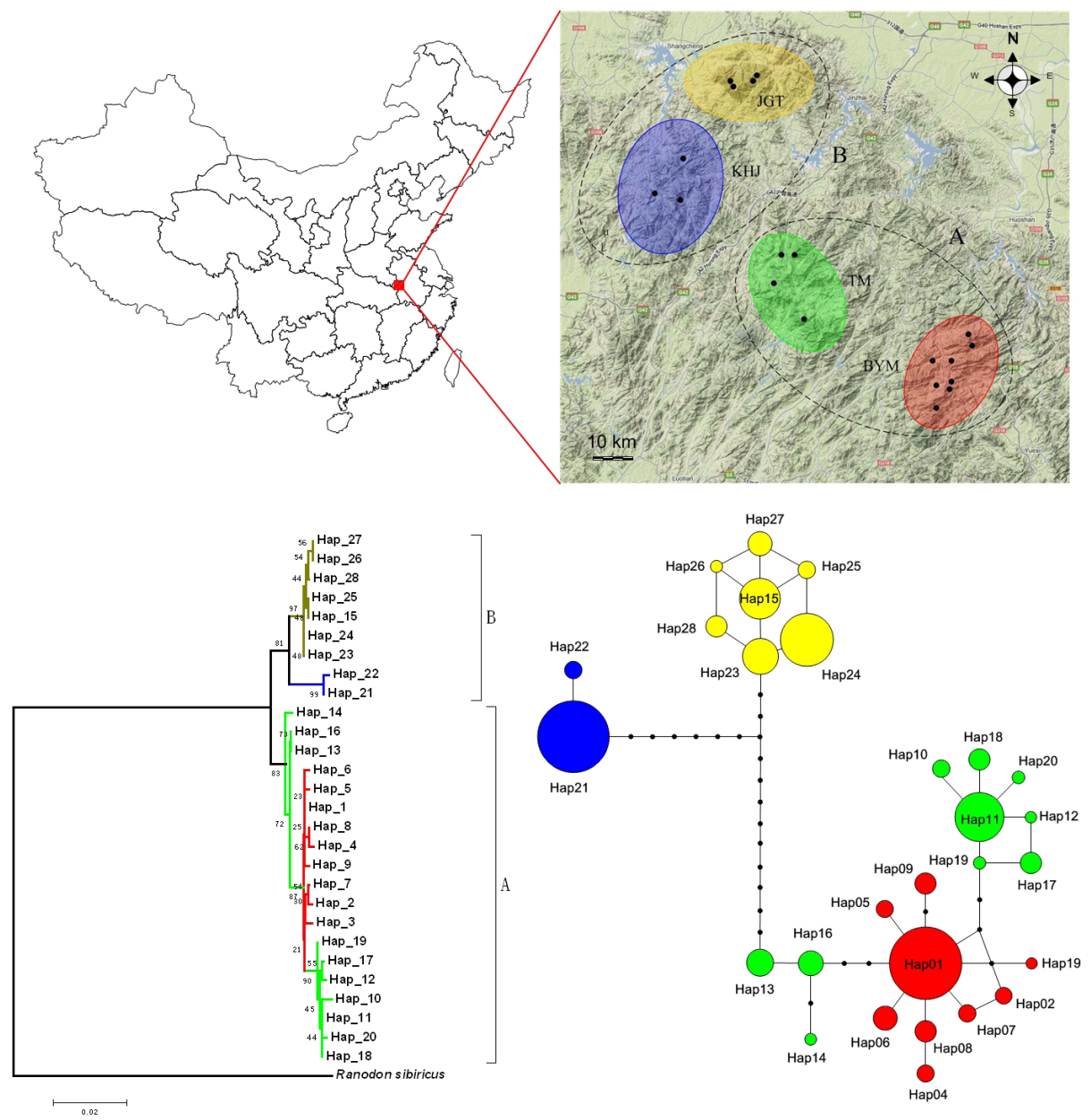

Figure 1. Geographic distribution of the 19 sampling sites and phylogenetic relationships of the 28 haplotypes found in Pachyhynobius shangchengensis. The geographical origin of haplotypes is indicated by different colors (red - BYM, green - TM, blue - KHJ, and yellow - JGT). Neighbor-joining tree showed the phylogenetic relationships among the 28 haplotypes. Figures on branches are the branch support values. Ranodon sibiricus is an outgroup. Minimum-spanning network connecting the 28 identified mitochondrial control region haplotypes based on statistical parsimony using NETWORK 4.6.1.0. The size of circles is roughly proportional to haplotype frequency. For population abbreviations, see legend to Table 1. 
Table 1. Sampling locality information, genetic diversity, growth parameter, and neutrality test for partial D-loop sequences in different population of Pachyhynobius shangchengensis.

\begin{tabular}{|c|c|c|c|c|c|c|c|}
\hline Population & $\mathrm{N}$ & $H$ & $h$ & $\pi$ & $g$ & Tajima D test & Fu Fs test \\
\hline 1. BYM & 54 & 9 & $5730 \pm 0.0782$ & $0.0011 \pm 0.0009$ & $3268.73 \pm 68$ & $-1.392(\mathrm{P}$ & $-4.836(\mathrm{P}$ \\
\hline 2. TM & 37 & 10 & $0.7853 \pm 0$ & $0.0050 \pm 0.0034$ & $-31.64 \pm 129.20$ & $0.378(\mathrm{P}=0.697)$ & $-0.01997(\mathrm{P}=0.554)$ \\
\hline 3. KHJ & 36 & 2 & $0.1079 \pm 0.0680$ & $0.0001 \pm 0.0003$ & $2925.68 \pm 2420.60$ & $-0.813(\mathrm{P}=0.5)$ & $-0.597(\mathrm{P}=0.124)$ \\
\hline 4. JGT & 48 & 7 & $0.7748 \pm 0.0361$ & $0.0017 \pm 0.0012$ & $2716.19 \pm 619.54$ & $1.247(\mathrm{P}=0.883)$ & $-1.248(\mathrm{P}=0.271)$ \\
\hline Total & 175 & 28 & $0.8969 \pm 0.0119$ & $0.0159 \pm 0.0080$ & - & $1.840(\mathrm{P}=0.975)$ & $2.38657(\mathrm{P}=0.770)$ \\
\hline
\end{tabular}

$\mathrm{N}=$ No. of sampling; $H=$ No. of haplotypes; $h=$ haplotype diversity; $\pi=$ nucleotide diversity; $g=$ growth parameter. BYM = Baimajian/Mingtangshan/Yaoluopin; TM = Tiantangzhai/Mazongling; KHJ = Kangwangzhai/ Huangbaishan/Jiufengjian; JGT = Jingangtai.

\section{Phylogeographic and population structure analyses}

A phylogenetic tree constructed using the sequences of the 28 haplotypes, indicated the existence of two main clades (labeled A and B in Figure 1), which were supported with a high level of confidence. Clade A comprised the 19 haplotypes from populations BYM and TM, while B comprised the 9 haplotypes from populations KHJ and JGT. Three haplotypes from population TM (Hap 13, Hap 14, and Hap 16) were at the root of clade A, and seven other haplotypes from TM and all nine haplotypes from BYM formed a subclade with a high confidence level. Overall, there was no clear monophyly corresponding to populations TM or BYM in clade A. The haplotypes in clade B formed two distinct monophyletic subclades corresponding to populations KHJ and JGT. Estimation of time to the most recent common ancestor (TMRCA) showed that all divergences within $P$. shangchengensis occurred in the Middle Pleistocene. The first divergence within P. shangchengensis occurred in $683 \mathrm{kya}(95 \% \mathrm{CI})$. The lineages within clade A diverged $448 \mathrm{kya}(95 \% \mathrm{CI})$ and those of clade B had diverged approximately 124 kya $(95 \% \mathrm{CI})$. The median-joining network suggested a similar phylogeographic pattern to the phylogenetic tree (Figure 1). The network consisted of three distinct haplogroups. The first haplogroup (in yellow) corresponded to population JGT (Figure 1), which was a compact type and contained haplotypes interconnected by a single mutation. The second haplogroup (in blue) was simple and contained two haplotypes connected by one-step mutation. The third was a complex haplogroup, comprising all the haplotypes from populations TM (green) and BYM (red). Haplotypes from population BYM were in a central position in this haplogroup, while the haplotypes from population TM were on the periphery. The range of differentiation among the four populations was 0.530-0.970 (Table 2). Remarkably, all the P values were significant $(<0.01)$. The pairwise $F_{\mathrm{ST}}$ value for populations KHJ and BYM $\left(F_{\mathrm{ST}}=0.970\right)$ was higher than for the other pairwise comparisons; the lowest value was for populations BYM and TM (0.530). Among the four populations, the mean genetic distance range was from 0.006 to 0.025 . Furthermore, the lowest genetic distance was detected between populations BYM and TM.

\begin{tabular}{|c|c|c|c|c|}
\hline & 1 & 2 & 3 & 4 \\
\hline 1. BYM & - & 0.006 & 0.025 & 0.020 \\
\hline 2. TM & $0.530^{*}$ & - & 0.025 & 0.021 \\
\hline 3. KHJ & $0.970^{*}$ & $0.905^{*}$ & - & 0.014 \\
\hline 4. JGT & $0.946^{*}$ & $0.879^{*}$ & $0.955^{*}$ & - \\
\hline
\end{tabular}

All tests were performed with 10,000 permutations. Significant tests are indicated $(* \mathrm{P}<0.0001)$. For population abbreviations, see legend to Table 1. 
AMOVA was used to relate the genetic variation to the geographic distance between the populations of $P$. shangchengensis. Most of the molecular variance was within the populations (60.25-65.79\%; Table 3). The highest variance that could be explained among populations relative to the entire sample occurred for the groupings: [BYM, TM] $[\mathrm{KHJ}][\mathrm{JGT}]\left(\Phi_{\mathrm{CT}}\right.$ $=0.179, \mathrm{P}=0.161 \pm 0.011$; Table 3 ). Mantel testing failed to identify a typical IBD model for $P$. shangchengensis; there was no correlation between $F_{\mathrm{ST}}$ and the geographic distance $(\mathrm{r}=$ $-0.0007 ; \mathrm{c}=-0.20, \mathrm{P}=0.53$ ).

Table 3. Hierarchical analysis of molecular variance (AMOVA) of control region sequence among Pachyhynobius shangchengensis samples.

\begin{tabular}{lcccccc}
\hline Groupings & $\begin{array}{c}\text { Among } \\
\text { groups }\end{array}$ & $\begin{array}{c}\text { Among populations } \\
\text { within groups }\end{array}$ & $\begin{array}{c}\text { Within } \\
\text { population }\end{array}$ & $\Phi_{\text {SC }}$ & $\Phi_{\text {ST }}$ & $\Phi_{\text {CT }}$ \\
\hline [BYM] [TM,KHJ,JGT] & -8.69 & 44.28 & 64.41 & $0.407(\mathrm{P}=0)$ & $0.356(\mathrm{P}=0)$ & $-0.087(\mathrm{P}=0.493)$ \\
{$[\mathrm{BYM}, \mathrm{TM}][\mathrm{KHJ}, \mathrm{JGT}]$} & -0.10 & 37.71 & 62.39 & $0.377(\mathrm{P}=0)$ & $0.376(\mathrm{P}=0)$ & $-0.001(\mathrm{P}=0.683)$ \\
{$[\mathrm{BYM}, \mathrm{TM}, \mathrm{KHJ}][\mathrm{JGT}]$} & -11.43 & 45.64 & 65.79 & $0.410(\mathrm{P}=0)$ & $0.342(\mathrm{P}=0)$ & $-0.114(\mathrm{P}=0.758)$ \\
{$[\mathrm{BYM}, \mathrm{TM}][\mathrm{KHJ}][\mathrm{JGT}]$} & 17.87 & 21.88 & 60.25 & $0.267(\mathrm{P}=0)$ & $0.398(\mathrm{P}=0)$ & $0.179(\mathrm{P}=0.161)$ \\
{$[\mathrm{BYM}][\mathrm{TM}, \mathrm{KHJ}][\mathrm{JGT}]$} & -19.29 & 55.67 & 63.62 & $0.467(\mathrm{P}=0)$ & $0.364(\mathrm{P}=0)$ & $-0.193(\mathrm{P}=0.669)$ \\
\hline
\end{tabular}

\section{Historical demographic analysis}

The mismatch distributions in the four populations were not decisively unimodal (Figure 2) and so the expansion model was rejected.

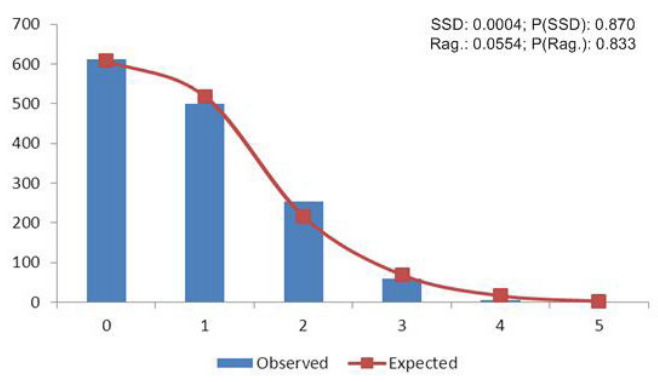

A

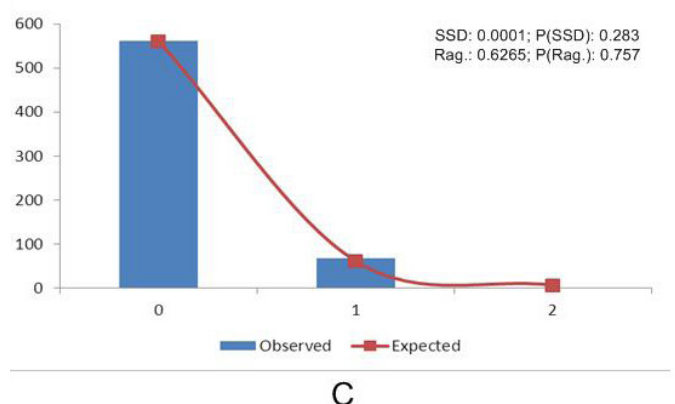

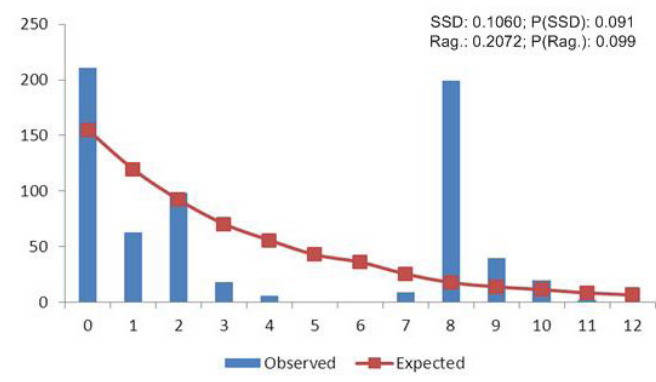

B

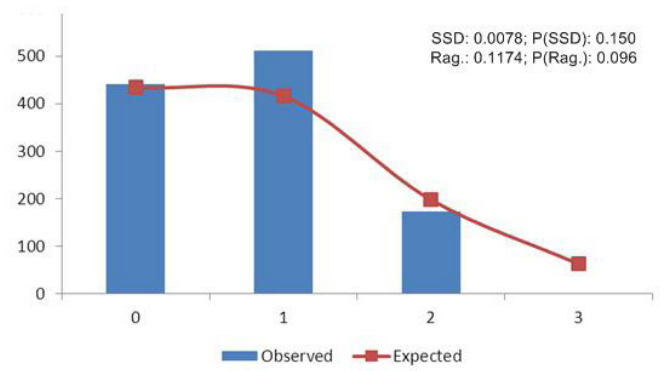

D

Figure 2. Mismatch distribution for each population of Pachyhynobius shangchengensis. A. B. C. and D. Mismatch distributions for the whole sample set, BYM, TM, KHJ, and JGT, respectively. SSD = sum of squared deviations; Rag = raggedness index. 
The population growth parameter $(g)$ from the maximum likelihood approach showed that all populations except population TM had undergone strong population expansion; TM had undergone a weak population contraction $(g=-31.64, \mathrm{SD}= \pm 129.20)$. In the neutrality test, none of the calculated values of the Tajima D test was statistically significant, although the values of populations BYM and KHJ were not neutral (Table 1). All values of the Fu Fs test were negative and only population BYM was statistically significant $(\mathrm{Fs}=-4.836, \mathrm{P}=0.003)$. Assessment of the BSPs indicated that the effective population size has remained stable for a long time ( $\sim 60 \mathrm{kya})$, until a recent population fluctuation ( $20 \mathrm{kya}$; Figure 3$)$. This population fluctuation may be closely related to the action of the last glacial maximum (LGM; 15-25 kyr).

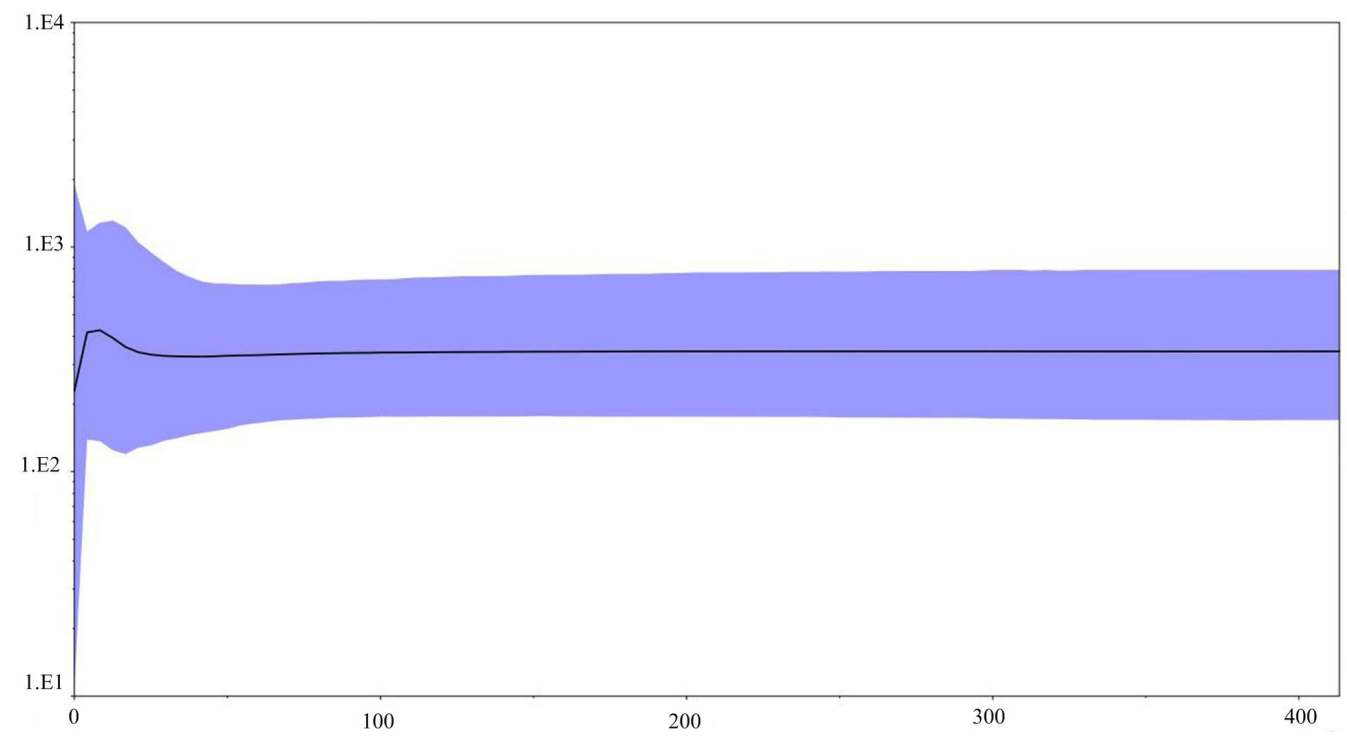

Figure 3. Bayesian skyline plot showing the historical demographic trend of Pachyhynobius shangchengensis. Time (x-axis) is reported in years and population size (y-axis) is measured as the product of effective population size per generation length.

\section{DISCUSSION}

\section{Genetic diversity}

According to the haplotype and genetic diversity data (Table 1), lower genetic variability was detected in extant populations of $P$. shangchengensis than has been found in some endangered Caudata, such as Andrias davidianus (Tao et al., 2005) and Plethodon vehiculum (Pelletier et al., 2011). In particular, only two haplotypes were detected in the 36 samples from population KHJ. Such low genetic diversity could be caused by decreases in population size, population extinction, genetic drift, and habitat loss (Allentoft and Brien, 2010); factors that have been discussed previously in relation to amphibians (Murphy et al., 2000). Furthermore, some studies have proposed that isolated populations may have low genetic variation (Ohnishi et al., 2007). Urban development has also been a major cause of fragmented habitats. The genetic diversity of four populations of red-backed salamander (Plethodon cinereus) was lower 
as a result of isolation due to habitat loss and fragmentation caused by roads, graveyards, and buildings (Noel et al., 2007). Even for some dispersed species, such as Rana temporaria, anthropogenic habitat destruction has reduced population density and genetic diversity (Johansson et al., 2005). The habitat of $P$. shangchengensis had been seriously reduced over the last decade, leading to marked reductions in population density and size (Cai, 2001; Xiong et al., 2009). Ten years of continuous monitoring in Yingshan (1989-1999) have shown that population size and density of $P$. shangchengensis have undergone a sustained decrease (Cai, 2001). The population decrease of $P$. shangchengensis has been attributed to the following reasons: 1) Loss and fragmentation of habitat through deforestation, land reclamation, construction of houses, tourism-related development, and other human activity; and 2) Excessive hunting. In Yaoluoping National Nature Reserve, the habitat of $P$. shangchengensis has become highly fragmented (Wang et al., 2009). Local residents often catch $P$. shangchengensis for food or as pets. Moreover, the larvae are often captured to be sold as Andrias davidianus larvae, which are of high commercial value (Xiong et al., 2009). Both factors have led to population decrease, localized extinction, and genetic drift; thus, causing further decrease in genetic diversity.

\section{Phylogeography and population divergence}

Low-elevation areas with unfavorable environmental conditions act as barriers separating populations on different mountains, thereby creating a 'sky island' situation. High levels of inter-population genetic divergence have often been observed in species that are restricted to montane habitats (Shepard and Burbrink, 2008, 2009). In the Dabie Mountains, P. shangchengensis is restricted to streams above $500 \mathrm{~m}$, which are separated by warmer, more xeric valleys (Cai, 2001; Xiong et al., 2009; Zhang et al., 2009). Four divergent lineages are present in these mountains, corresponding to four areas of distribution. It seems that xeric ridges and low-elevation streams prevent or restrict gene flow, as has been reported in studies of Plethodon ouachitae and Plethodon fourchensis (Shepard and Burbrink, 2008, 2009). The phylogenetic analysis presented in this study supports the existence of a distinct split between northern (JGT and KHJ) and southern populations (BYM and TM). Furthermore, haplotypes from populations JGT and KHJ aggregate in distinct subclades within the northern lineage (clade B) and form haplogroups in network estimation analysis (Figure 1). This may be due to the long-term isolation of populations JGT and KHJ. In contrast, populations BYM and TM do not form two distinct subclades. Evidence of admixture between these southern populations was found in both the phylogenetic and network analysis (Table 3 and Figure 1). Unfortunately, no strong evidence was found that proves that this admixture arose from renewed contact between these populations. Our data suggest that either the separation of these populations was more recent, or that ancestral population size was larger, requiring a longer time to diverge to monophyly (Hudson and Coyne, 2002). The hypothesis that niche conservatism drives diversification in montane species predicts that such lineages will be separated by unsuitable environmental conditions (Shepard and Burbrink, 2008 , 2009). Based on our analyses, several lines of evidence suggest the existence of a distinct phylogeographic outlier from range of this species, and the existence of major discontinuities in its geographic distribution. AMOVA suggests that most of the variation exists within the populations, which is also supported by $F_{\mathrm{ST}}$ values. This was also supported by the lack of overlapping haplotypes among the four populations. Our results provide new insight, from a previously unstudied region, into the phylogeography of narrowly endemic species and the process by which 'sky islands' become more diverse, particularly for species that are environmentally sensitive. 


\section{Historical demography}

Environmental shifts in montane regions, induced by climatic changes during the Pleistocene glacial cycles, have resulted in population divergence on different mountains (Hewitt, 1996). The divergence dates from coalescent simulations are consistent with this hypothesis, indicating that lineage diversification in P. shangchengensis occurred during the Pleistocene, with the TMRCA of all populations dating to 683 kya (Middle Pleistocene). The demographic trend inferred from BSP shows that the population size increased around $25 \mathrm{kyr}$ after a prolonged phase of demographic stability (of nearly $600 \mathrm{kyr}$ ) and reached its maximum at approximately 10 kya (Figure 3). The LGM was around 18 kya and the glaciation began to recede around 12 kya (Maley and Brenaca, 1998); considering the intrinsic inaccuracy of the molecular clock (Ayala, 1997; Gibbons, 1998), it seems likely that the glaciation contributed significantly to the population growth of $P$. shangchengensis. The apparent population fluctuation was also supported by other approaches, such as the $g$ parameter and two neutrality tests (Table 1). The geographic distribution of singleton mutations, corresponding to the star-like haplogroup in the network, may be the consequence of demographic expansions (RamosOnsins and Rozas, 2002). During the Pleistocene, several climatic changes occurred (Clark et al., 2006). In these glacial cycles, cold glacial periods lasted for most of each cycle and the interglacial periods lasted for only 10-20 kyr (Clark et al., 2006). During the interglacial period, climatic conditions in unglaciated lower latitudes were generally warmer and wetter, leading to expansion of deciduous forest (Denniston et al., 1999). The same regions were generally colder and drier in the glacial periods, leading to contraction of deciduous forest and a dominance of conifers (Jackson et al., 2000). The longer glacial and interglacial periods, together with the increased amplitude of climatic fluctuations (i.e., higher extremes of temperature and precipitation), would have provided both more opportunity for $P$. shangchengensis to disperse to nearby unoccupied mountains (during interglacial periods) and more time for lineages isolated on different mountains to diverge (during the glacial periods).

\section{Implications for conservation}

Severe regional decreases in $P$. shangchengensis have led to concerns about the long-term viability of this species (Cai, 2001; Xiong et al., 2009; Zhang et al., 2009). Here we present a fourpoint argument as to why we believe $P$. shangchengensis have undergone severe regional decreases in recent times, including a combination of obvious anthropogenic factors and life-history traits.

Firstly, a small effective population size is directly associated with the loss of genetic diversity through genetic drift and inbreeding (Schmeller and Merila, 2007). Furthermore, lower population density is connected with effective population size (Levitan, 1991).

Secondly, habitat fragmentation may have had one of the most important roles. Habitat fragmentation directly leads to a decrease in population size. Moreover, localized extinction increases the risk of inbreeding depression (Cushman, 2006). A long history of low diversity may have led to increases in deleterious alleles by natural selection (Allentoft and Brien, 2010).

Thirdly, anthropogenic impacts are an irrefutable factor. The destruction and fragmentation of habitat, pollution, fatalities on roads, and over-harvesting for human consumption are direct causes of population decrease (Hels and Buchwald, 2001; Warkentin et al., 2009).

Lastly, the limited capacity for dispersal in this species, compared to most other ver- 
tebrates, may also have resulted in population decrease. Amphibians are often described as highly site-philopatric and demonstrate poor dispersal capabilities, even in natural environments (Allentoft and Brien, 2010). This phenomenon is commonly explained by a dependence on moisture. Previous studies have focused on behavior and physiology (Chen, 1991; Zhang et al., 2006) instead of conservation. The most effective strategy for the conservation of $P$. shangchengensis in the Dabie Mountains may be to use genetic data from distributed areas to prioritize and protect them. Evolutionarily significant units and management units can be identified through phylogenetic and network studies (De Guia and Saitoh, 2007). Although this species is narrowly distributed, this study supports the existence of four MUs, defined by the geographical populations reported in this study (BMY, TM, KHJ, and JGT), and three corresponding evolutionarily significant units (with BMY and TM as a single unit).

\section{ACKNOWLEDGMENTS}

Research supported by the National Nature Science Foundation of China (NSFC, \#30800116, \#31272332), the Anhui University Young \& Core Teachers' Training Program, and the Open Foundation of Jiangsu Key Laboratory for Biodiversity and Biotechnology (\#B200712-1). We are grateful to Faguang Pu, Yangguo Fang, Yaoxian Li, Wenge Wang, Wenliang Zhou, Bin Liu, Junlin Chen, Tian-Ma National Nature Reserve, and Yaoluoping National Nature Reserve for their help in sampling and laboratory work.

\section{REFERENCES}

Allentoft ME and Brien JO (2010). Global amphibian declines, loss of genetic diversity and fitness: A Review. Diversity 2: 47-71.

Ayala FJ (1997). Vagaries of the molecular clock. Proc. Natl. Acad. Sci. U. S. A. 94: 7776-7783.

Bandelt HJ, Forster P and Rohl A (1999). Median-joining networks for inferring intraspecific phylogenies. Mol. Biol. Evol. 16: 37-48.

Cai S (2001). The ecological observation and resources protection of Pachyhynobius shangchengensis. J. Cent. China Normal Univ. (Nat. Sci.) 35: 203-205.

Chen B (1991). The Amphibian and Reptilian Fauna of Anhui. Anhui Science \& Technology Publishing House, Hefei.

Church SA, Kraus JM, Mitchell JC, Church DR, et al. (2003). Evidence for multiple Pleistocene refugia in the postglacial expansion of the eastern tiger salamander, Ambystoma tigrinum tigrinum. Evolution 57: 372-383.

Clark PU, Archer D, Pollard D, Blumd JD, et al. (2006). The Middle Pleistocene transition: characteristics, mechanisms, and implications for long-term changes in atmospheric $\mathrm{pCO}_{2}$. Quaternary Sci. Rev. 25: 3150-3184

Clarke KR and Gorley RN (2001). PRIMER Version 5: User Manual/Tutorial. Primer-E Ltd., Plymouth, 91.

Cushman SA (2006). Effects of habitat loss and fragmentation on amphibians: A review and prospectus. Biol. Conserv. 128: $231-240$.

De Guia APO and Saitoh T (2007). The gap between the concept and definitions in the Evolutionarily Significant Unit: the need to integrate neutral genetic variation and adaptive variation. Ecol. Res. 22: 604-612.

DeChaine EG and Martin AP (2005). Historical biogeography of two alpine butterflies in the Rocky Mountains: broadscale concordance and local-scale discordance. J. Biogeogr. 32: 1943-1956.

Denniston RF, Gonzalez LA, Semken HA, Asmeromb Y, et al. (1999). Integrating stalagmite, vertebrate, and pollen sequences to investigate Holocene vegetation and climate change in the southern Midwestern United States. Quaternary Res. 52: 381-387.

Drummond AJ and Rambaut A (2007). BEAST: Bayesian evolutionary analysis by sampling trees. BMC Evol. Biol. 7: 214.

Excoffier L, Laval G and Schneider S (2005). Arlequin (version 3.0): an integrated software package for population genetics data analysis. Evol. Bioinform. Online 1: 47-50.

Fei L, Qu W and Wu S (1985). Description of a new genus and species of Hynobiidae of China. Zool. Res. 6: 399-404. 
Gibbons A (1998). Calibrating the mitochondrial DNA clock. Science 279: 38-39.

Hels T and Buchwald E (2001). The effect of road kills on amphibian populations. Biol. Conserv. 99: 331-340.

Hewitt GM (1996). Some genetic consequences of ice ages, and their role in divergence and speciation. Biol. J. Linn. Soc. 58: $247-276$.

Hewitt GM (2004). Genetic consequences of climatic oscillations in the quaternary. Phil. Trans. Roy. Soc. Lond. B Biol. Sci. 359: 183-195.

Hudson RR and Coyne JA (2002). Mathematical consequences of the genealogical species concept. Evolution 56: 15571565.

Jackson ST, Webb RS, Anderson KH, Overpeck JT, et al. (2000). Vegetation and environment in eastern North America during the last glacial maximum. Quaternary Sci. Rev. 19: 489-508.

Johansson M, Primmer CR, Sahlsten J and Merilã J (2005). The influence of landscape structure on occurrence, abundance and genetic diversity of the common frog, Rana temporaria. Global Change Biol. 11: 1664-1679.

Levitan DR (1991). Influence of body size and population density on fertilization success and reproductive output in a free-spawning invertebrate. Biol. Bull. 181: 261-268.

Librado P and Rozas J (2009). DnaSP v5: a software for comprehensive analysis of DNA polymorphism data. Bioinformatics 25: 1451-1452.

Lind AJ, Spinks PQ, Fellers GM and Shaffer HB (2011). Rangewide phylogeography and landscape genetics of the Western U.S. endemic frog Rana boylii (Ranidae): implications for the conservation of frogs and rivers. Conserv. Genet. 12: 269-284.

Maley J and Brenaca P (1998). Vegetation dynamics, palaeoenvironments and climatic changes in the forests of western Cameroon during the last 28,000 years B.P. Rev. Palaeobot. Palynol. 99: 157-187.

Murphy RW, Fu J, Upton DE, Lema TD, et al. (2000). Genetic variability among endangered Chinese giant salamanders, Andrias davidianus. Mol. Ecol. 9: 1539-1547.

Noel S, Ouellet M, Galois P and Lapointe FJ (2007). Impact of urban fragmentation on the genetic structure of the eastern red-backed salamander. Conserv. Genet. 8: 599-606.

Nylander JAA (2004). MrModeltest v2: Evolutionary Biology Centre. Uppsala University, Olmstead.

Ohnishi N, Saitoh T, Ishibashi Y and Oi T (2007). Low genetic diversities in isolated populations of the Asian black bear (Ursus thibetanus) in Japan, in comparison with large stable populations. Conserv. Genet. 8: 1331-1337.

Palsbøll PJ, Berube M and Allendorf FW (2007). Identification of management units using population genetic data. Trends Ecol. Evol. 22: 11-16.

Pelletier TA, Duffield DA and DeGrauw EA (2011). Rangewide phylogeography of the Western Red-Backed Salamander (Plethodon vehiculum). Northwest. Nat. 92: 200-210.

Rambaut A and Drummond AJ (2007). Tracer v1.4. Available at [http://beast.bio.ed.ac.uk/Tracer]. Accessed November $21,2012$.

Ramos-Onsins SE and Rozas J (2002). Statistical properties of new neutrality tests against population growth. Mol. Biol. Evol. 19: 2092-2100.

Sambrook JEF, Fritsch EF and Maniatis T (1989). Molecular Cloning: A Laboratory Manual. 2nd edn. Cold Spring Harbor Laboratory Press, Cold Spring Harbor.

Schmeller DS and Merila J (2007). Demographic and genetic estimates of effective population and breeding size in the amphibian Rana temporaria. Conserv. Biol. 21: 142-151.

Shepard DB and Burbrink FT (2008). Lineage diversification and historical demography of a sky island salamander, Plethodon ouachitae, from the Interior Highlands. Mol. Ecol. 17: 5315-5335.

Shepard DB and Burbrink FT (2009). Phylogeographic and demographic effects of Pleistocene climatic fluctuations in a montane salamander, Plethodon ouachitae. Mol. Ecol. 18: 2243-2262.

Steinfartz S, Veith M and Tautz D (2000). Mitochondrial sequence analysis of salamandra taxa suggests old splits of major lineages and postglacial recolonizations of central Europe from distinct source populations of Salamandra salamandra. Mol. Ecol. 9: 397-410.

Swofford DL (2003). PAUP*. Phylogenetic Analysis Using Parsimony (*and Other Methods). Version 4. Sinauer Associates, Sunderland.

Tamura K, Dudley J, Nei M and Kumar S (2007). MEGA4: Molecular Evolutionary Genetics Analysis (MEGA) software version 4.0. Mol. Biol. Evol. 24: 1596-1599.

Tao FY, Wang XM, Zheng HX and Fang SG (2005). Genetic structure and geographic subdivision of four populations of the Chinese giant salamander (Andrias davidianus). Zool. Res. 26: 162-167.

Thompson JD, Higgins DG and Gibson TJ (1994). CLUSTAL W: improving the sensitivity of progressive multiple sequence alignment through sequence weighting, position-specific gap penalties and weight matrix choice. Nucleic Acids Res. 22: 4673-4680. 
Veness C (2010). Calculate Distance, Bearing and More Between Latitude/Longitude Points. Available at [http://www. movable-type.co.uk/scripts/latlong.html]. Accessed November 21, 2012.

Wang S, Bao F, Qi Y and Xu J (2009). Resource distribution and habitat selection of Pachyhynbius shangchengsis in Yaoluoping Nature Reserve. Amino Acids Biotic Resour. 31: 4-7.

Warkentin IG, Bickford D, Sodhi NS and Bradshaw CJ (2009). Eating frogs to extinction. Conserv. Biol. 23: 1056-1059.

Wiens JJ (2004). Speciation and ecology revisited: phylogenetic niche conservatism and the origin of species. Evolution 58: 193-197.

Xiong J, Sun P, Zhu L and Liu X (2009). A Specific Hynobiidae in China: Pachyhynobius shangchengensis. Anim. Husbandry Feed. Sci. 6: 133-134.

Zhang B, Shi W, Zhao K, Xu X, et al. (2009). Preliminary investigation of Pachyhynobius shangchengensis distribution and its injury and malformation phenomenon. Herpetol. Sin. 12: 346-351.

Zhang P, Chen YQ, Zhou H, Liu YF, et al. (2006). Phylogeny, evolution, and biogeography of Asiatic salamanders (Hynobiidae). Proc. Natl. Acad. Sci. U. S. A. 103: 7360-7365. 